\title{
Aggregation Behavior of Amphiphilic PAMAM-Based Hyperbranched Polymer in the Presence of Conventional Small Molecular Surfactants
}

\author{
Hui Yang ${ }^{1}$, Danfeng Yu ${ }^{1}$, Hui Wang ${ }^{1}$, Quan Xie ${ }^{2}$, Jiazhong $\mathrm{Wu}^{2}$, Jinben Wang ${ }^{1 *}$ \\ ${ }^{1}$ Key Laboratory of Colloid, Interface and Chemical Thermodynamics, Institute of Chemistry, \\ The Chinese Academy of Sciences, Beijing, China \\ ${ }^{2}$ State Key Laboratory of Enhanced Oil Recovery, Research Institute of Petroleum Exploration and \\ Development of CNPC, Beijing, China \\ Email: ${ }^{*}$ jbwang@iccas.ac.cn
}

Received April 25, 2013; revised May 25, 2013; accepted June 25, 2013

Copyright (C) 2013 Hui Yang et al. This is an open access article distributed under the Creative Commons Attribution License, which permits unrestricted use, distribution, and reproduction in any medium, provided the original work is properly cited.

\begin{abstract}
Hyperbranched polymer composed of G1 polyamidoamine (PAMAM) and branched with poly (propylene oxide) (PPO)-block-poly (ethylene oxide) (PEO) was investigated to interact with sodium dodecyl sulfate (SDS) and di-dodecyl dimethyl ammonium bromide (DDAB), respectively, by the methods of turbidity titration and analysis, rheology measurements, dynamic light scattering (DLS) and transmission electron microscopy (TEM). It was noticeable that even at extremely low concentration of SDS (even far from the critical micelle concentration (cmc)), the system exhibits high turbidity, indicating that SDS molecules can insert into cationic amine groups and hydrophobic microenvironment, resulting in the formation of polymer-SDS complexes with large size. At the SDS concentration range of below $0.1 \mathrm{mM}$, the turbidity and cloud point (CP) temperature of the system keep almost invariable, mostly because of the repulsion between SDS molecules and the complexes. And, therefore, the size of the mixed aggregates retains almost constant. In the case of vesicle system of DDAB, the aggregates are in the size of $100 \mathrm{~nm}-200 \mathrm{~nm}$ and $500 \mathrm{~nm}-3000$ $\mathrm{nm}$ at the concentrations of $30 \mathrm{mM}$ and $100 \mathrm{mM}$, respectively. However, in the mixture of hyperbranched polymer with DDAB, by comparison, the size is smaller in a binary system than that of in DDAB system. So it is reasonable to infer that DDAB molecules remove from multilamellar vesicles of DDAB to the hydrophobic microenvironment of hyperbranched polymer aggregates, with the addition of the hyperbranched polymer. It leads to the destruction of the gel-like conformation in DDAB system, leading to the shear thinning of the mixture and, as a result, the viscoelastic character of the system is lost in a large degree.
\end{abstract}

Keywords: Hyperbranched Polymer; Aggregate Conformation; Complex

\section{Introduction}

Hyperbranched polymers are highly branched and three-dimensional macromolecules with dentritic architecture and long repeat units [1]. Due to their unique physical and chemical properties, hyperbranched polymers have gained significant attention from both academia and industry [2,3]. Various examples of PAMAM-based polymers functionalized with rigid hydrophobic periphery, chromophoric groups, or binding metal ions have been investigated intensively these years [4,5]. Little attention, however, was paid to the aggregation behavior of PAMAM molecules branched with amphiphilic segments

${ }^{*}$ Corresponding author.
[6], especially to understand their solution behavior or with the addition of surfactants [7-11].

Thus far, surfactant-hyperbranched polymer interactions have been concerned, which are important in many potential application fields, such as drug delivery vehicles, cosmetic, food, and paint industries $[12,13]$. The mixed systems of PAMAM and surfactant in aqueous solution have been investigated by several researchers [14-17]. It showed that the aggregation between the hyperbranched polymer and surfactant is significantly dependent upon the nature of surface groups, or ionic surfactant head groups [18]. Surface activity and hydrophobicity of the surfactants are enhanced upon interaction with PAMAM dendrimers, and the apparent hydrody- 
namic radius largely increases with such interactions [19-22].

In the investigation of salt effect and $\mathrm{pH}$ response on the series of polymers with poly(propylene oxide) (PPO)-block-poly(ethylene oxide) (PEO), we found that the hyperbranched amphiphiles are very surface active, of low aggregation concentration, and the aggregation behavior and aggregates conformation are sensitive to $\mathrm{pH}$ and salt [23-25]. With the increase of $\mathrm{pH}$ value, the hyperbranched polymer solution transformed from turbid to transparent, due to the protonation process of PAMAM; with the addition of inorganic salts, the hydration water molecules preferentially move from coordination shells of hyperbranched polymer molecules to the hydration layer of salt molecules; and the benzene ring of organic salt has a significant effect in increasing the size of complex aggregates, owing to penetrating into the hydrophobic core of aggregates. As a further study, the work has been extended to investigate the molecular interactions in the micellization of amphiphilic polymers and conventional surfactants. The mixtures are characterized by turbidity titration, DLS and TEM methods. The different aggregation properties of it in aqueous solutions are evaluated in absence as well as presence of additives (surfactants). Nowadays, many investigations have been made on the supramolecular structures and interactions of PAMAM molecules, but little has been reported on the relationship between amphiphilic PAMAM-based polymer and surfactant. In order to further understand such interactions, we investigated the interplay between this type of polymer and sodium dodecyl sulfate (SDS) through examining the change in turbidity and aggregation properties, and the transition in rheology and aggregation behavior of the mixed system of the polymer and didodecyldimethylammonium bromide (DDAB).

\section{Experimental Section}

\subsection{Materials}

Chemicals were obtained from Beijing Chemical Reagents Company and were of analytical grade. Solutions were prepared with triply distilled water.

\subsection{Synthesis and Characterization}

The route of synthesis on hyperbranched polymer and DDAB followed the previous work $[23,26]$.

\subsection{Measurements}

Turbidimetric Titration. The turbidity of hyperbranched polymer solution with titrated SDS was measured at $450 \mathrm{~nm}$ using a Brinkman PC920 probe colorimeter equipped with a thermostated water-circulating bath. The cloud point (CP) of the system at different conditions was obtained from the break of $100-T \%$ (transmittance) vs. temperature curves.

Rheology. The rheological properties of samples were measured at $25.00^{\circ} \mathrm{C} \pm 0.01^{\circ} \mathrm{C}$ with a Thermo Haake Rheo Stress 300 rheometer (cone and plate geometry of $35 \mathrm{~mm}$ in diameter with the cone gap equal to $0.105 \mathrm{~mm}$ ). The range of frequency scanning is $0.1-20 \mathrm{rad} \cdot \mathrm{s}^{-1}$.

Dynamic Light Scattering (DLS). DLS measurement was introduced, employing an LLS spectrometer (ALV/ SP-125) with a multi- $\tau$ digital time correlator (ALV$5000)$. Light $(\lambda=632.8 \mathrm{~nm})$ from a solid-state He-Ne laser $(22 \mathrm{~mW})$ was used as the incident beam. The scattering angle was selected to $90^{\circ}$ and the correlation function was analyzed with the Contin method. Solutions were filtrated through $0.45 \mu \mathrm{m}$ millipore to leach dust, and were laid until stabilization before measurements.

Transmission Electron Microscope (TEM). Negative-staining (with uranyl acetate aqueous solution of 1 $\mathrm{wt} \%$ ) and freeze-fracture (FF) techniques were used for TEM sample preparation. Fracturing and replication were carried out in a high-vacuum freeze-etching system (Balzers BAF-400D). The samples were imaged under a Hitachi H800 electron microscope.

\section{Results and Discussion}

\subsection{Aggregation Behavior of the Mixture of Hyperbranched Polymer and SDS}

At the temperature of $15^{\circ} \mathrm{C}$, the turbidity curve of hyperbranched polymer (at $1 \mathrm{wt} \%$ ) with titrated SDS is shown in Figure 1, which exhibits that turbidity value ascends with the concentration of SDS increasing firstly, indicating that there may be strong interaction between hyperbranched polymer and SDS molecules. As the concentration of SDS comes to about $0.024 \mathrm{mM}$, the turbidity value reaches the peak. When it is higher than $0.11 \mathrm{mM}$

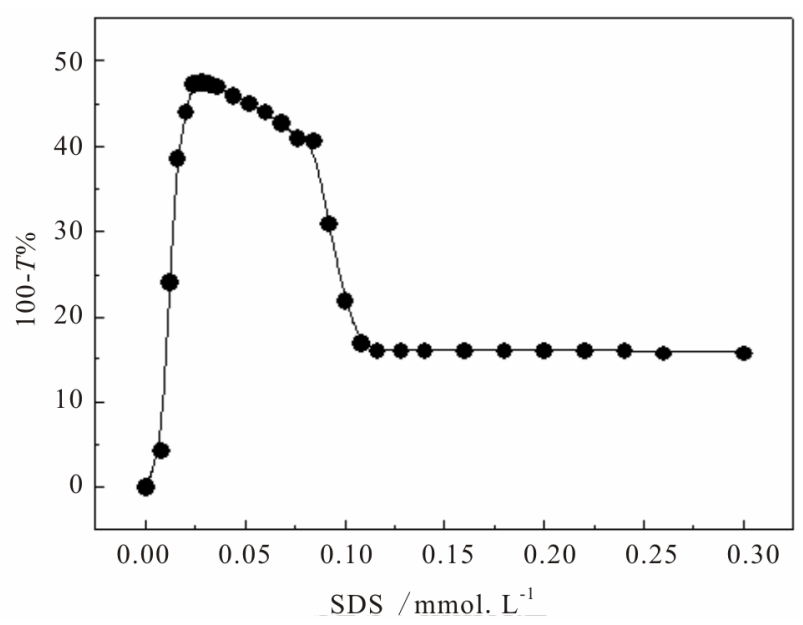

Figure 1. Turbidity curve of hyperbranched polymer solution with the titration of SDS. 
(around 1\% of cmc of SDS), the turbidity value keeps a constant of around $15 \%$, inclining to an unchangeable state.

Turbidity vs. temperature curves of hyperbranched polymer with SDS at different concentrations and the cloud point (CP) temperatures are shown in Figure 2 and Table 1, separately. As the concentration of SDS increases, $\mathrm{CP}$ of the mixture decreases at first (in the concentration range of $0-0.05 \mathrm{mM}$ ), and then tends to be almost invariable around $24^{\circ} \mathrm{C}(0.1 \mathrm{mM}-0.5 \mathrm{M})$. The results show that the hyperbranched polymer interacts with SDS even at very low concentration (such as 0.01 and $0.05 \mathrm{mM}$ ); while $\mathrm{CP}$ varies little with added SDS at higher concentration $(\geq 0.1 \mathrm{mM})$, which matches well with the deduction from turbidity titration experiments.

With the addition of SDS, $R_{\mathrm{h}}$ of aggregates increases to 220.2 and $396.1 \mathrm{~nm}$ at the SDS concentration of 0.01 and $0.05 \mathrm{mM}$ (Figure 3 and Table 1), respectively, indicating that the hyperbranched polymer-SDS complex forms and leads to the formation of bigger aggregates. This result is in well agreement with that of turbidity and $\mathrm{CP}$ measurements, in which the turbidity value increases at the same SDS concentration range. In this case, SDS molecules could insert into cationic amine groups [27] and hydrophobic microenvironment [28] as designed and

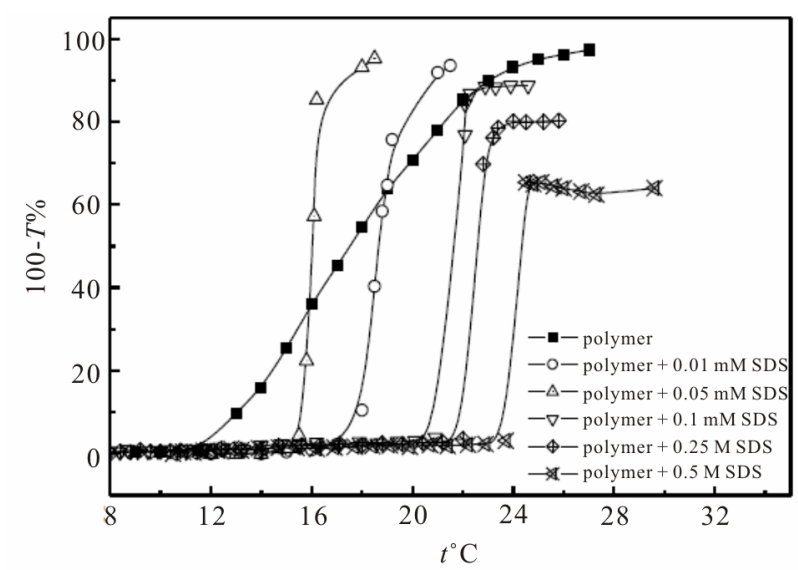

Figure 2. Cloud point (CP) curves of mixed system of SDS and hyperbranched polymer.

Table 1. Apparent hydrodynamic radius $\left(R_{h}\right)$ of the aggregates and CP temperature of the mixed system.

\begin{tabular}{cccc}
\hline \multicolumn{2}{c}{ Mixed system } & CP $\left({ }^{\circ} \mathrm{C}\right)$ & $R_{\mathrm{h}}(\mathrm{nm})$ \\
\cline { 1 - 2 }$C_{\text {Hyperbranched polymer }}$ & $C_{\mathrm{SDS}}$ & & 54.9 \\
\multirow{2}{*}{$1 \%$} & 0 & 22.5 & 220.2 \\
& $0.01 \mathrm{mM}$ & 19.2 & 396.1 \\
\hline & $0.05 \mathrm{mM}$ & 16.2 & 375.5 \\
& $0.1 \mathrm{mM}$ & 22.9 & $3.1 ; 379.1$ \\
& $0.25 \mathrm{M}$ & 23.4 & $4.8 ; 370.3$ \\
\hline
\end{tabular}

drawn in Figure 4, which leads to the enlargement of aggregate size. As the concentration of SDS is higher than $0.1 \mathrm{mM}$ corresponding with the invariable turbidity, the $R_{\mathrm{h}}$ value remains around $370 \mathrm{~nm}$, mainly because of the repulsion between SDS molecules and that in hyperbranched polymer-SDS complexes [29]. As the concentration keeps increasing (up to 0.25 and $0.5 \mathrm{M}$ ), there is an $R_{\mathrm{h}}$ distribution of $3-5 \mathrm{~nm}$ promoted from the self-aggregation of SDS molecules, or the aggregation of multimolecular SDS and monomolecular hyperbranched polymer [30,31].

Through TEM method the molecular aggregation behavior was examined as shown in Figures 5(a)-(f). The aggregates of hyperbranched polymer are around $100 \mathrm{~nm}$

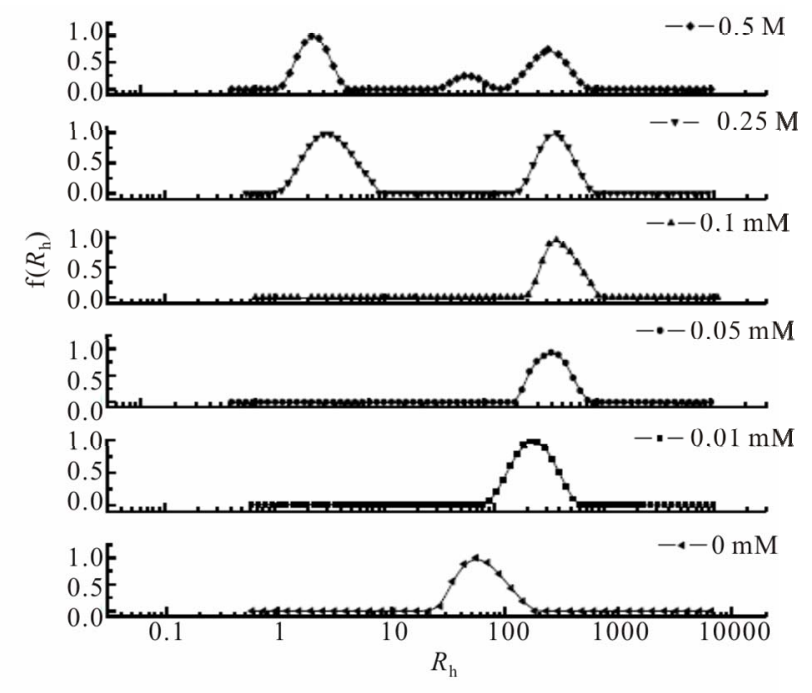

Figure 3. DLS results of the binary system at the SDS concentration range of $0-0.5 \mathrm{M}$.

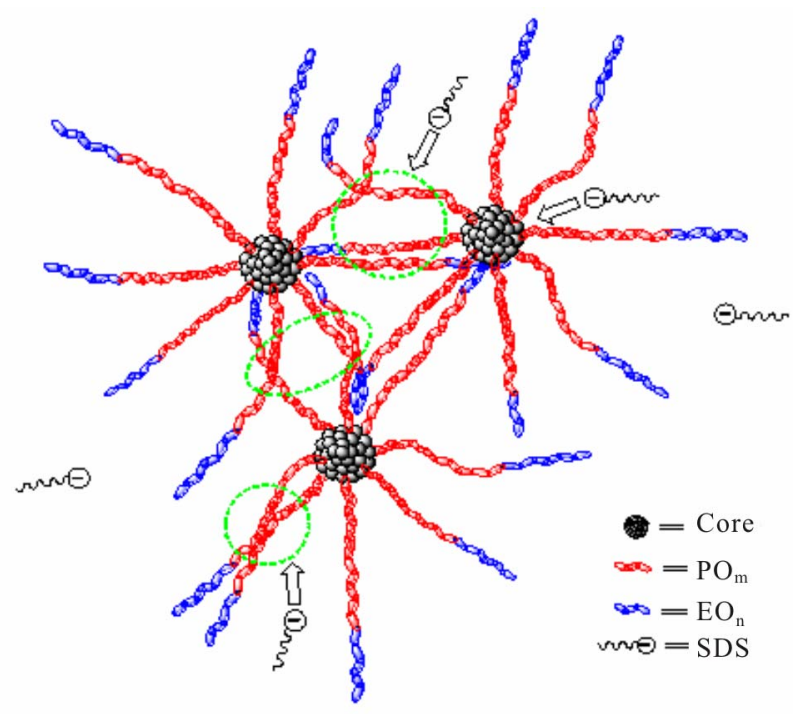

Figure 4. Scheme of interaction between hyperbranched polymer and SDS molecules. 

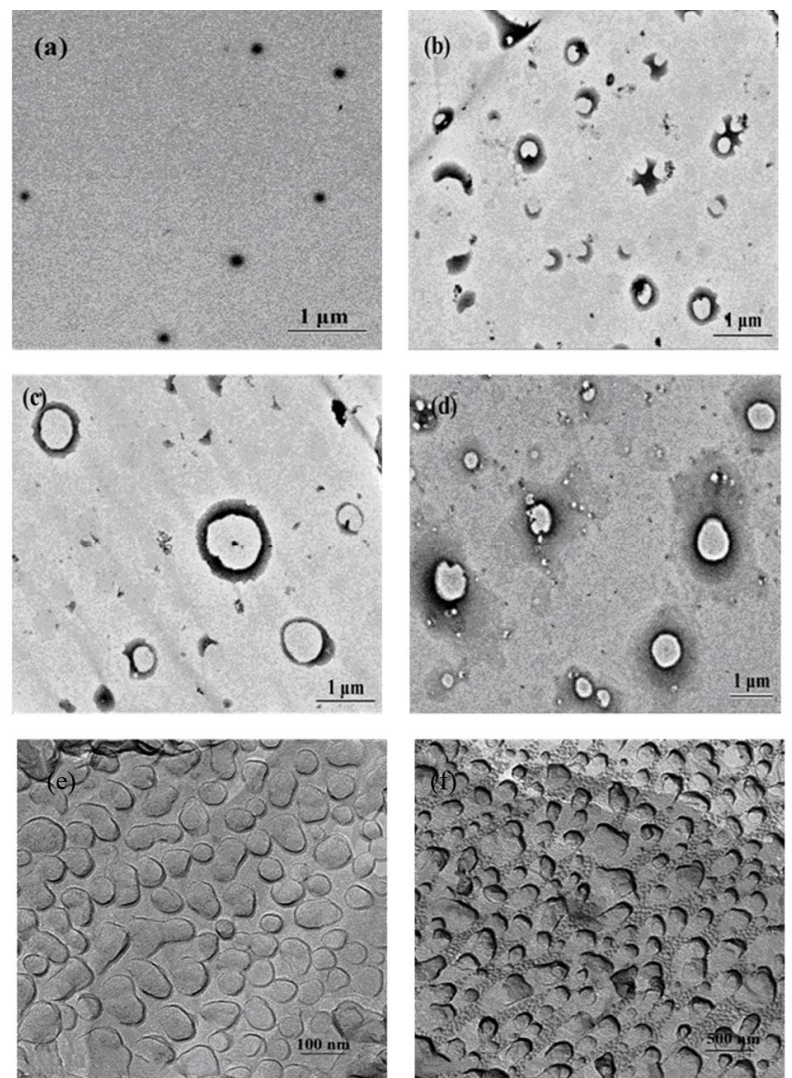

Figure 5. TEM photos of the mixed system of hyperbranched polymer interacting with SDS: (a) 1\% hyperbranched polymer (Negative-staining); $0.01 \mathrm{mM}$ SDS + \% hyperbranched polymer (Negative-staining); (c) $0.05 \mathrm{mM}$ SDS + 1\% hyperbranched polymer (Negative-staining); (d) 0.25 M SDS + 1\% hyperbranched polymer (Negative-staining) (e) 1\% hyper-branched polymer (FF); (f) $0.01 \mathrm{mM}$ SDS + 1\% hyper-branched polymer (FF).

as observed through negative-staining (Figure 5(a)) and freeze-fracture (Figure 5(e)) techniques. The size of aggregates grows to 400,800 and $800 \mathrm{~nm}$ with the addition of SDS at $0.01 \mathrm{mM}, 0.05 \mathrm{mM}$, and $0.25 \mathrm{M}$, respectively, as shown in Figures 5(b)-(d) and (f). Moreover, with higher content SDS, there are smaller aggregates in the system, which is in agreement with that from DLSmethod.

\subsection{Rheological Properties of the Mixed System of Hyperbranched Polymer and DDAB}

From Figures 6(a) and (b), vivid bilayer vesicle can be observed, and the size becomes bigger ranging from 100 - $200 \mathrm{~nm}$ to $500-3000 \mathrm{~nm}$ with the concentration increase from $30 \mathrm{mM}$ to $100 \mathrm{mM}$. Figure 6(c) shows a collection of unilamellar vesicles with an average size around $100 \mathrm{~nm}$, while Figures 6(d)-(f) show typical multilamellar aggregates in the size of $1-3 \mu \mathrm{m}$, which exhibits that giant multilamellar vesicles coexist with smaller unilamellar ones and some of the multilamellar
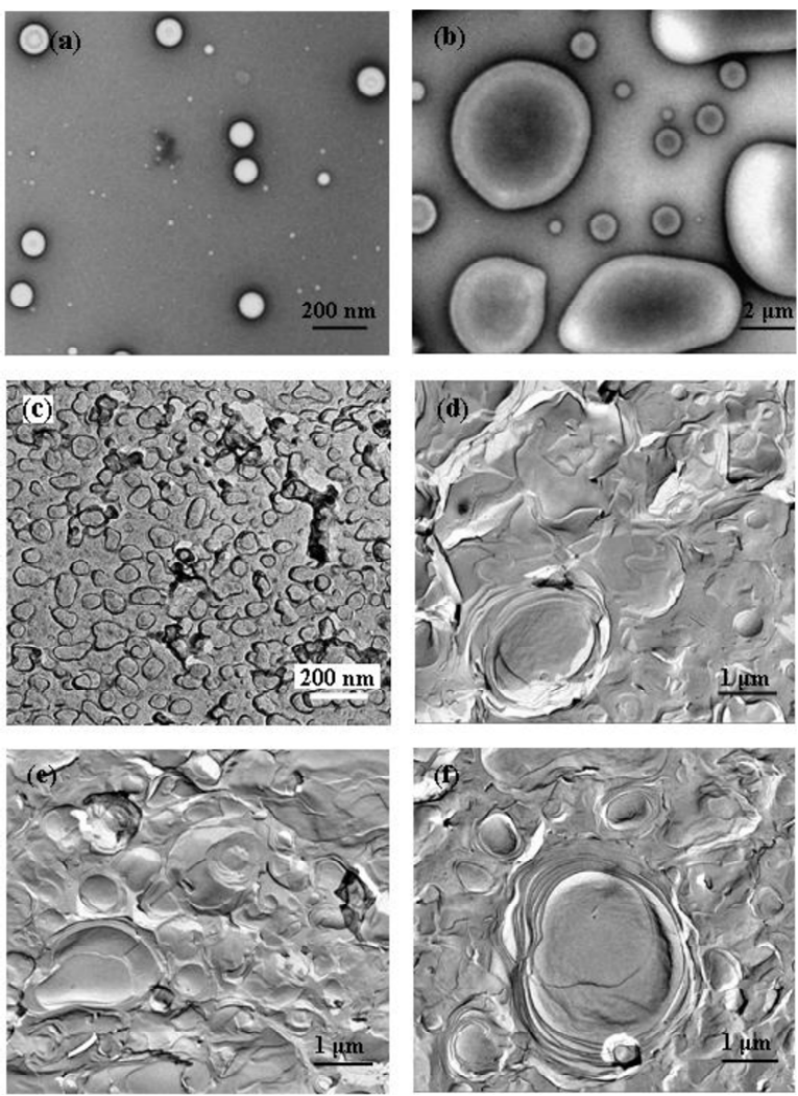

Figure 6. TEM images of DDAB aggregates at different concentrations: (a) $30 \mathrm{mM}$ (Negative-staining); (b) $100 \mathrm{mM}$ (Negative-staining); (c) 30 mM (FF); (d) 60 mM (FF); (e) 80 mM (FF); (f) 100 mM (FF).

aggregates are curved structures like "onion" shells. Almost each vesicle is surrounded by other ones and locates in a cage, which is expected from packing constraints forcing the multilayered structures to occur. From the "cage" it can not escape by a simple diffusion process without deformation of its "shells", and therefore, the system has a viscoelastic property under deformation [32]. The mixed system with hyperbranched polymer and DDAB (at different concentrations) is investigated through TEM measurements shown in Figures 7(a)-(f). The size of aggregates is about $100 \mathrm{~nm}$ and $1 \mu \mathrm{m}$ with the added DDAB at the concentration from $30 \mathrm{mM}$ to 100 $\mathrm{mM}$, which is smaller than that of DDAB system. And the structure transforms from multilamella to unilamella, indicating that the "shell-cage" conformation is mostly destroyed (because there is interaction between DDAB and hyperbranched polymer).

The shear rheology curves of the mixed system are shown in Figure 8(b). The viscosity of the mixture decreases from 1000 to $100 \mathrm{~s}^{-1}$, which is lower than that of DDAB system, because the gel-like conformation of DDAB system transforms to unilamella vesicle system. Furthermore, it comes from the interaction between hy- 

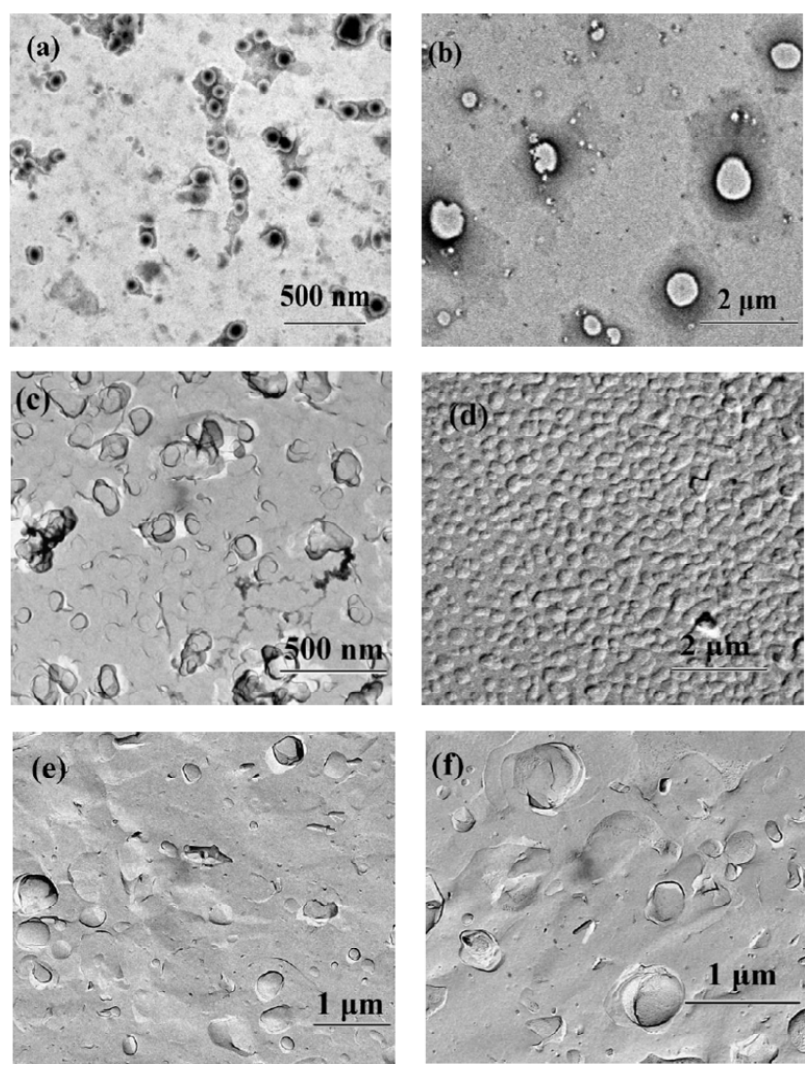

Figure 7. TEM photos of binary system of DDAB and hyperbrabched polymer at differert conditions: (a) $1 \%$ hyperbranched polymer + $30 \mathrm{mM}$ DDAB (Negative-staining); (b) $1 \%$ hyperbranched polymer $+100 \mathrm{mM}$ DDAB (Negative-staining); (c) $1 \%$ hyperbranched polymer $+30 \mathrm{mM}$ DDAB (FF); (d) 1\% hyperbranched polymer + $60 \mathrm{mM}$ DDAB (FF); (e) 1\% hyper-branched polymer (FF); (f) 0.01 mM SDS + 1\% hyper-branched polymer (FF).

perbranched polymer and DDAB, as well as the self-aggregation of DDAB molecules.

Plots of rheological parameters $G^{\prime}(\omega)$ and $G^{\prime \prime}(\omega)$ versus angular frequency $\omega$ are shown in Figure 9. Generally, the storage modulus $G^{\prime}$ gives a measure of elastic properties of the material and the loss modulus $G^{\prime \prime}$ reflects a certain degree of viscosity [33]. Within the studied frequency range, $G^{\prime}$ and $G^{\prime \prime}$ modules exhibit frequency independence at 80 and $100 \mathrm{mM}$ and shows typical gel-like behavior (see the curves a and b). $G^{\prime \prime}$ has higher values than $G^{\prime \prime}$, indicating that the system has a weak viscoelastic characteristic at these concentrations. However, at lower concentration (lower than 80 $\mathrm{mM}$ ), the solution is of weaker viscoelastic with lower values of $G^{\prime}$ and $G^{\prime \prime}$ (see the curve c). $G^{\prime \prime}$ value is bigger than that of $G^{\prime}$ at the $\omega$ range of $0.1-1 \mathrm{~s}^{-1}$, but smaller over $1-10 \mathrm{~s}^{-1}$, which indicates that $G^{\prime}$ and $G^{\prime \prime}$ become frequency-dependent. Hence, the colloid solution becomes higher viscoelastic and the relation between $G^{\prime}$ and $G^{\prime \prime}$ changes from frequency depen-
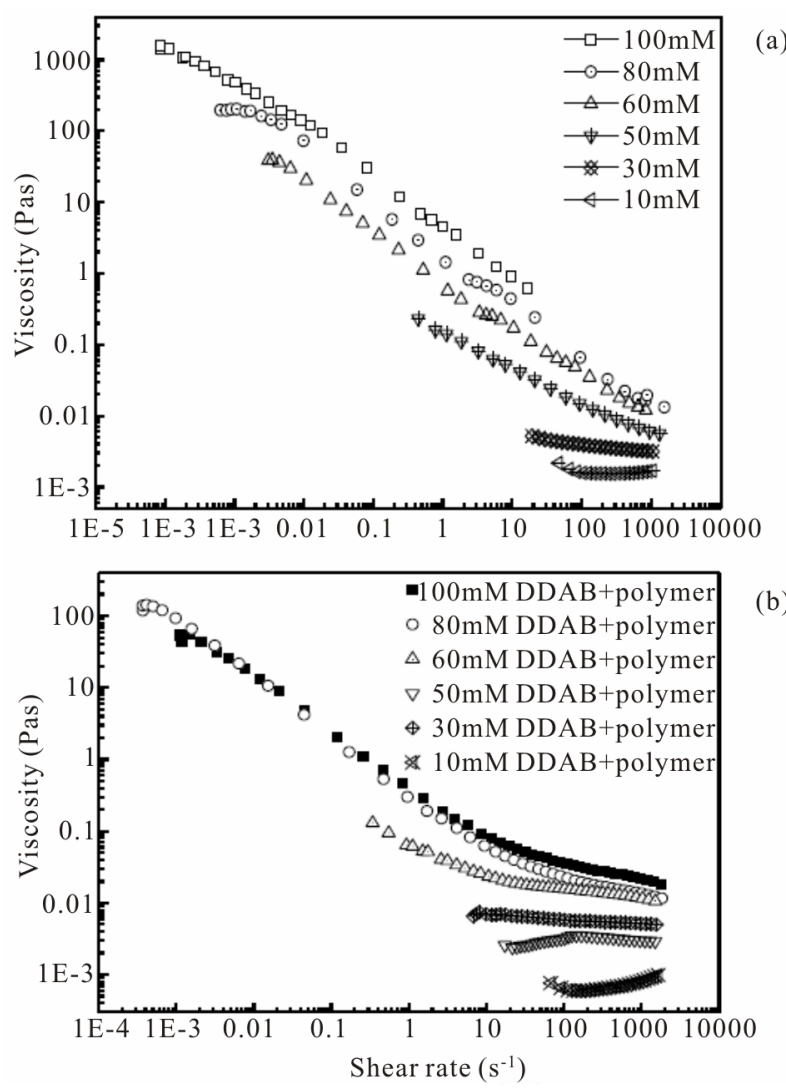

Figure 8. Steady shear rheology curves: (a) DDAB; (b) DDAB + 1\% hyperbrabched polyer.

dence to independence, mostly due to the aggregate conformation changing with the increase of concentration. However, with the addition of hyperbranched polymer, the system can not be detected the values of $G^{\prime}$ and $G^{\prime \prime}$ vs. $\omega$, so the binary system does not possess viscoelasticity, indicating that the gel-like configuration is destructed and some DDAB molecules remove from multilamellar vesicles of DDAB to the hydrophobic microenvironment of hyperbranched polymer (Figure 10), and therefore the unilamellar vesicles of DDAB and the hyper- branched polymer-DDAB complexes form to a large extent.

\section{Conclusions}

Through measurements of turbidity analysis, DLS and TEM, the interaction between hyperbranched polymer and SDS, including aggregation behavior and turbidity property of the mixed system, was investigated. With SDS even at low concentration such as far from CMC, there is strong molecular interaction in polymer-SDS system. The aggregates become larger with the addition of SDS, because SDS molecules can insert into amine group and hydrophobic microenvironment. When the concentration of SDS is higher than $0.1 \mathrm{mM}$, the size of 


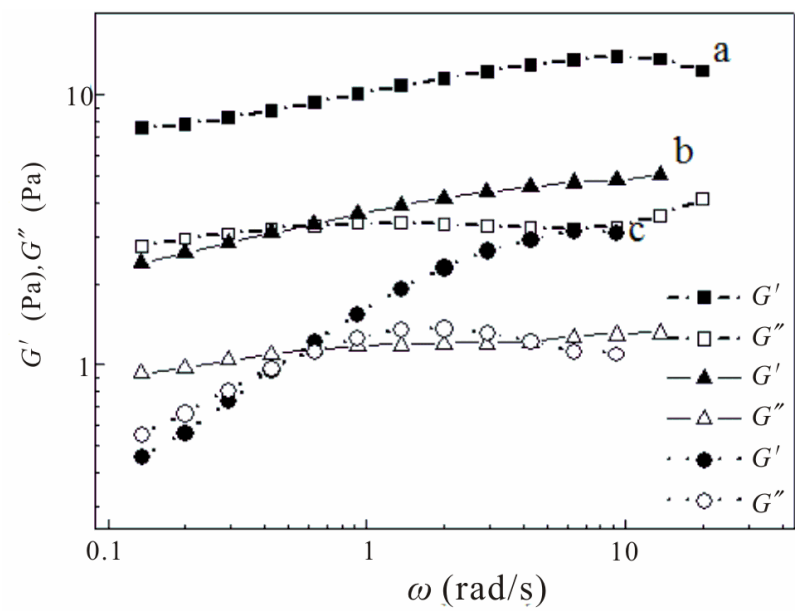

Figure 9. Storage modulus $\left(G^{\prime}\right)$ and loss modulus $\left(G^{\prime \prime}\right)$ as a function of angular frequency $(\omega)$ at: a $60 \mathrm{mM}$, b $80 \mathrm{mM}$ and c $100 \mathrm{mM}$.
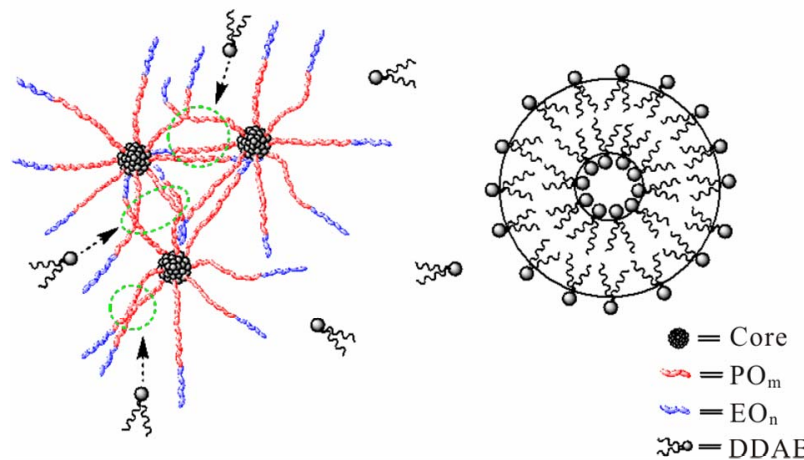

Figure 10. Scheme of interaction between hyperbranched polymer and DDAB molecules.

aggregates keeps constant due to the repulsion among SDS molecules and hyperbranched polymer-SDS complexes. More SDS molecules scarcely lead aggregates continue growing but induce the self aggregates of SDS or multi-molecules of SDS aggregating with monomolecular hyperbranched polymer.

By comparison, the viscoelasticity of DDAB in the presence of hyperbranched polymer was investigated by the methods of steady and dynamic state rheological properties, the detection and analysis of aggregation behavior. The results showed that the gel-like conformation is broken in the presence of hyperbranched polymer. And then, the size of aggregates decreases from $100-200 \mathrm{~nm}$ and $500-3000 \mathrm{~nm}$ (in DDAB system at the concentration from $30 \mathrm{mM}$ to $100 \mathrm{mM}$ ) to $100 \mathrm{~nm}$ and $1 \mu \mathrm{m}$ (in the mixed system of hyperbranched polymer and DDAB; DDAB at the same concentration range), and the aggregates morphology transforms from multilamella to unilamellar. It induces the viscosity to decrease largely after shearing, and the mixture nearly shows no viscoelastic property.

\section{Acknowledgements}

The authors are thankful for the Key Project of Chinese National Programs for Fundamental Research and Development (2012CB723601) and the Important National Science and Technology Specific Project of China (2011ZX05024-004-03).

\section{REFERENCES}

[1] C. Gao and D. Yan, "Hyperbranched Polymers: From Synthesis to Applications," Progress in Polymer Science, Vol. 29, No. 3, 2004, pp. 183-275. doi:10.1016/j.progpolymsci.2003.12.002

[2] P. J. Flory, "Molecular Size Distribution in Three Dimensional Polymers. VI. Branched Polymers Containing A-R-B $\mathrm{B}_{\mathrm{f}-1}$ Type Units," Journal of the American Chemical Society, Vol. 74, No. 11, 1952, pp. 2718-2723. doi:10.1021/ja01131a008

[3] C. R. Yates and W. Hayes, "Synthesis and Applications of Hyperbranched Polymers," European Polymer Journal, Vol. 40, No. 7, 2004, pp. 1257-1281. doi:10.1016/j.eurpolymj.2004.02.007

[4] E. K. Yeow, K. P. Ghiggino, J. N. Reek, M. J. Crossley, A. W. Bosman, A. P. Schenning and E. W. Meijer, "The Dynamics of Electronic Energy Transfer in Novel Multiporphyrin Functionalized Dendrimers: A Time-Resolved Fluorescence Anisotropy Study," The Journal of Physical Chemistry B, Vol. 104, No. 12, 2000, pp. 2596-2606. doi:10.1021/jp993116u

[5] M. S. Diallo, W. Arasho, J. H. Johnson Jr. and W. A. Goddard III, "Dendritic Chelating Agents. 2. U(VI) Binding to Poly(amidoamine) and Poly(propyleneimine) Dendrimers in Aqueous Solutions," Environmental Science \& Technology, Vol. 42, No. 5, 2008, pp. 15721579. doi:10.1021/es0715905

[6] X. Xin, G. Y. Xu, Z. Q. Zhang, Y. J. Chen and F. Wang, "Aggregation Behavior of Star-Like PEO-PPO-PEO Block Copolymer in Aqueous Solution," European Polymer Journal, Vol. 43, No. 7, 2007, pp. 3106-3111. doi:10.1016/j.eurpolymj.2007.04.005

[7] A. Sh. King, I. K. Martin and L. J. Twyman, "Synthesis and Aggregation of Amine-Cored Polyamidoamine Dendrons Synthesised without Invoking a Protection/Deprotection Strategy," Polymer International, Vol. 55, 2006, p. 798-807.

[8] J. Wang, C. Q. Li, J. Li and J. Z. Yang, "Demulsification of Crude Oil Emulsion Using Polyamidoamine Dendrimers," Separation Science and Technology, Vol. 42, No. 9, 2007, pp. 2111-2120. doi:10.1080/01496390701241857

[9] A. G. Escudero, M. A. Azagarsamy, N. Theddu, R. W. Vachet and S. Thayumanavan, "Selective Peptide Binding Using Facially Amphiphilic Dendrimers," Journal of the American Chemical Society, Vol. 130, No. 33, 2008, pp. 11156-11163. doi:10.1021/ja803082v

[10] X. F. Li, T. Imae, D. Leisner and M. A. Lopez-Quintela, "Lamellar Structures of Anionic Poly(amido amine) Dendrimers with Oppositely Charged Didodecyldimethylammonium Bromide," The Journal of Physical Chemis- 
try B, Vol. 106, No. 47, 2002, pp. 12170-12177. doi:10.1021/jp0209260

[11] L. Porcar, Y. Liu, R. Verduzco, K. L. Hong, P. D. Butler, L. J. Magid, G. S. Smith and W. R. Chen, "Structural Investigation of PAMAM Dendrimers in Aqueous Solutions Using Small-Angle Neutron Scattering: Effect of Generation," The Journal of Physical Chemistry B, Vol. 112, No. 47, 2008, pp. 14772-14778. doi:10.1021/jp805297a

[12] P. Alexandridis, "Amphiphilic Copolymers and Their Applications, " Current Opinion in Colloid \& Interface Science, Vol. 1, No. 4, 1996, pp. 490-501. doi:10.1016/S1359-0294(96)80118-X

[13] Y. Cheng, Y. Li, Q. Wu and T. Xu, "New Insights into the Interactions between Dendrimers and Surfactants by Two Dimensional NOE NMR Spectroscopy," The Journal of Physical Chemistry B, Vol. 112, No. 40, 2008, pp. 12674-12680.

[14] M. F. Ottaviani, P. Andechaga, N. J. Turro and D. A. Tomalia, "Model for the Interactions between Anionic Dendrimers and Cationic Surfactants by Means of the Spin Probe Method," The Journal of Physical Chemistry $B$, Vol. 101, No. 31, 1997, pp. 6057-6065. doi:10.1021/jp963271y

[15] K. Esumi, R. Saika, M. Miyazaki and K. Torigoe, "Interactions of Poly(amidoamine)dendrimers Having Surface Carboxyl Groups with Cationic Surfactants," Colloids and Surfaces A: Physicochemical and Engineering Aspects, Vol. 166, No. 1-3, 2000, pp. 115-121. doi:10.1016/S0927-7757(99)00451-3

[16] M. Miyazaki, K. Torigoe and K. Esumi, "Interactions of Sugar-Persubstituted Poly(amidoamine) Dendrimers with Anionic Surfactants," Langmuir, Vol. 16, No. 4, 2000, pp. 1522-1528. doi:10.1021/la990347j

[17] K. Esumi, H. Chiba, H. Mizutani, K. Shoji and K. Torigoe, "Physicochemical Properties of Aqueous Mixed Solutions of Sugar-Persubstituted Poly(amidoamine)dendrimers and Anionic Surfactants," Colloids and Surfaces A: Physicochemical and Engineering Aspects, Vol. 179, No. 1, 2001, pp. 103-109. doi:10.1016/S0927-7757(00)00722-6

[18] M. S. Bakshi and R. Sood, "Cationic Surfactant-Poly(amido amine) Dendrimer Interactions Studied by Krafft Temperature Measurements," Colloids and Surfaces A: Physicochemical and Engineering Aspects, Vol. 233, 2003, pp. 203-210.

[19] D. Astruc, E. Boisselier and C. Ornelas, "Dendrimers Designed for Functions: From Physical, Photophysical, and Supramolecular Properties to Applications in Sensing, Catalysis, Molecular Electronics, Photonics, and Nanomedicine," Chemical Reviews, Vol. 110, No. 4, 2010, pp. 1857-1959. doi:10.1021/cr900327d

[20] K. Esumi, T. Chiba, H. Mizutami, K. Shoji and K. Torigoe, "Interactions of Poly(amidoamine)dendrimers Having Surface Carboxyl Groups with Cationic Surfactants," Colloids and Surfaces A: Physicochemical and Engineering Aspects, Vol. 166, No. 1-3, 2000, pp. 115121. doi:10.1016/S0927-7757(99)00451-3

[21] K. Esumi, T. Chiba, H. Misutani, K. Shoji and K. Torigoe,
"Physicochemical Properties of Aqueous Mixed Solutions of Sugar-Persubstituted Poly(amidoamine)dendrimers and Anionic Surfactants," Colloids and Surfaces A: Physicochemical and Engineering Aspects, Vol. 179, No. 1, 2001, pp. 103-109. doi:10.1016/S0927-7757(00)00722-6

[22] T. Yoshimura, J. Fukai, H. Mizutani and K. J. Esumi, Colloid Interface Sci., Vol. 255, 2002, p. 428.

[23] H. Yang, Y. Han, S. Yang, W. Zhang, G. Tan, Y. Wang and J. Wang, "Aggregation Behaviour of a Novel Series of Polyamidoamine-Based Dendrimers in Aqueous Solution," Supramolecular Chemistry, Vol. 21, No. 8, 2009, pp. 754-758. doi:10.1080/10610270902980598

[24] H. Yang, S. Yang, X. Wu, J. Zhou and J. Wang, "Syntheses and Surface Properties of a Novel Series of Polyamidoamine Branched with PPO-PEO Dendrimers," Acta Physico-Chimica Sinica, Vol. 25, 2009, pp. 1806-1810.

[25] W. Zhang, G. Dong, H. Yang, J. Sun, J. Zhou and J. Wang, "Synthesis, Surface and Aggregation Properties of a Series of Amphiphilic Dendritic Copolymers," Colloids and Surfaces A: Physicochemical and Engineering Aspects, Vol. 348, No. 1-3, 2009, pp. 45-48. doi:10.1016/j.colsurfa.2009.06.029

[26] R. Zana, M. Benrraou and R. Rueff, "Alkanediyl-. alpha.,.omega.-bis(dimethylalkylammonium bromide) Surfactants. 1. Effect of the Spacer Chain Length on the Critical Micelle Concentration and Micelle Ionization Degree," Langmuir, Vol. 7, No. 6, 1991, pp. 1072-1075. doi:10.1021/la00054a008

[27] M. S. Bakshi and A. Kaura, "Fluorescence Studies of Interactions of Ionic Surfactants with Poly(amidoamine) Dendrimers," Colloids and Surfaces A: Physicochemical and Engineering Aspects, Vol. 284, No. 2, 2005, pp. 680686.

[28] T. Yoshimura, J. Fukai, H. Mizutani and K. Esumi, "Physicochemical Properties of Quaternized Poly(amidoamine) Dendrimers with Four Octyl Chains," Journal of Colloid and Interface Science, Vol. 255, No. 2, 2002, pp. 428-431.

[29] M. S. Bakshi, A. Kaura, R. Sood, G. Kaur, T. Yoshimura, K. Torigoe and K. Esumi, "Topographical and Photophysical Properties of Poly(amidoamine) Dendrimers with Ionic Surfactants," Colloids and Surfaces A: Physicochemical and Engineering Aspects, Vol. 266, No. 1-3, 2005, pp. 181-190. doi:10.1016/j.colsurfa.2005.06.059

[30] Y. Cheng, Y. Li, Q. Wu and T. Xu, "New Insights into the Interactions between Dendrimers and Surfactants by Two Dimensional NOE NMR Spectroscopy," The Journal of Physical Chemistry B, Vol. 112, No. 40, 2008, pp. 12674-12680. doi:10.1021/jp804954j

[31] K. Esumi, R. Saika, M. Miyazaki, K. Torigoe and Y. Koide, "Interactions of Poly(amidoamine)dendrimers Having Surface Carboxyl Groups with Cationic Surfactants," Colloids and Surfaces A: Physicochemical and Engineering Aspects, Vol. 166, No. 1-3, 2000, pp. 115121. doi:10.1016/S0927-7757(99)00451-3

[32] H. Hoffmann, C. Thunig, P. Schmiedel and U. Munkert, "Surfactant Systems with Charged Multilamellar Vesicles and Their Rheological Properties," Langmuir, Vol. 10, No. 11, 1994, pp. 3972-3981. doi:10.1021/la00023a013 
[33] G. Montalvo, M. Valiente and A. Khan, "Shear-Induced Topology Changes in Liquid Crystals of the Soybean
Lecithin/DDAB/Water System," Langmuir, Vol. 23, No. 21, 2007, pp. 10518-10524. doi:10.1021/la701539f 